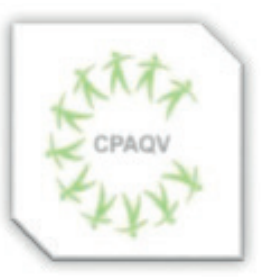

ISSN: 2178-7514
ARTIGO DE REVISÃO

\section{CAPACIDADES FÍSICAS NA PRÁTICA DO MUAY THAI}

\section{Physical Capabilities in Muay Thai Practice}

Robson Felix dos Santos ${ }^{1}$; Thiago Leite do Carmo Leite ${ }^{1}$; Bráulio Nascimento Lima ${ }^{1,2}$; Mariela de Santana Maneschy ${ }^{3}$; Renato Silveira de Assis Junior ${ }^{2}$; Klebson da Silva Almeida ${ }^{2,3}$; Ricardo Pablo Passos ${ }^{2,4}$; Guanis de Barros Vilela Junior ${ }^{2,4}$

Vol. 13 | No. 3| Ano 2021

\title{
RESUMO
}

O muay thai, é uma arte marcial milenar que vem ganhando espaço entre as atividades físicas mais praticadas nos últimos tempos. Por ser uma atividade física voltada para o público em geral, sua procura só cresce. Sua prática traz muitos benefícios à saúde do corpo e da mente, exige disciplina, postura, autocontrole e melhora o condicionamento físico, promovendo um desenvolvimento de suas capacidades físicas. As principais capacidades físicas são a agilidade, a flexibilidade e a força, que se fazem necessárias para a precisão e melhor desenvolvimento dos golpes. Assim, o presente projeto de pesquisa tem como objetivo o estudo teórico sobre o muay thai e sua relação com a melhora do condicionamento físico, bem como o estudo das capacidades físicas e sua contribuição na busca das pessoas pelo seu melhor resultado. Trata-se de uma pesquisa bibliográfica, que será realizada através de livros, artigos disponíveis em plataformas e periódicos diversos.

Palavras-chave: Muay thai, condicionamento físico, capacidades físicas.

\begin{abstract}
Muay thai is an ancient martial art that has been gaining space among the most practiced physical activities in recent times. As it is a physical activity aimed at the general public, its demand only grows. Its practice brings many benefits to the health of the body and mind, requires discipline, posture, self-control and improves physical conditioning, promoting the development of your physical capacities. The main physical capacities are agility, flexibility and strength, which are necessary for the precision and better development of strokes. Thus, the present research project aims at the theoretical study of muay thai and its relationship with the improvement of physical conditioning, as well as the study of physical capacities and their contribution in the search for people for their best result. This is a bibliographic search, which will be carried out through books, articles available on various platforms and journals.
\end{abstract}

Keywords: Muay thai, physical conditioning, physical capacities.

\footnotetext{
1 Faculdade Conhecimento e Ciência - FCC

2 Núcleo de Pesquisas em Biomecânica Ocupacional e Qualidade de Vida - NPBOQV

3 Universidade da Amazônia - UNAMA

4 Universidade Metodista de Piracicaba - UNIMEP
}

Autor de correspondência

Bráulio Nascimento Lima - brauliolima@outlook.com

DOI: $10.36692 / \mathrm{v} 13 \mathrm{n} 3-03 \mathrm{R}$ 


\section{INTRODUÇÃO}

O muay thai, também conhecido como boxe tailandês, surgiu acerca de dois mil anos na Tailândia. Sua origem teve muita influência do Kung Fu chinês, também conhecido como boxe chinês, porém ao longo do tempo ocorreram alterações significativas ${ }^{(1)}$.

Segundo a Confederação Brasileira de Muay Thai $\left(\mathrm{CBMT}^{2}{ }^{(2)}\right.$, em 1979, Nelio Naja introduziu o Muay Thai no Brasil, na época, mais conhecido como Boxe Tailandês, onde reuniu um grupo de faixas pretas de TaeKwondo, entre eles os Mestres Luiz Alves e Flávio Molina. Em 1980, foi fundada a primeira associação de Muay Thai, onde tinha como Presidente o Grão Mestre Flávio Molina; em, 1981 aconteceu o primeiro campeonato Interestadual no Rio de Janeiro no Bérro Dágua onde hoje é academia Nobre Arte, entre Rio de Janeiro vs Curitiba, onde Rio de Janeiro foi a campeã; ainda em 1981 o Fundador e Presidente da CBMT Grão Mestre Luiz Alves fazia a sua estreia como lutador de Muay Thai no desafio Rio Curitiba, onde venceu a final por $\operatorname{pontos}^{(2)}$.

$\mathrm{Na}$ década de 80, começa a nascer a primeira turma de atletas do Grão Mestre Luiz Alves, entre eles estava o Mestre Artur Mariano ${ }^{(2)}$. Em, 1987 foi fundada a primeira Federação da História do Brasil de Muay Thai, a Federação Carioca de Muay Thai, fundada pelo Grão Mestre Luiz Alves; o Muay Thai vinha crescendo de uma forma muita forte e sólida ${ }^{(2)}$.
Sua prática oferece inúmeros benefícios para o corpo e para a mente e pode ser realizada pelo público em geral ${ }^{(3)}$. Justamente por ser uma arte marcial que possibilita o uso de todas os membros é conhecida mundialmente como "a arte das oito armas", já que se caracteriza pelo uso combinado da técnica e da força dos membros do corpo humano. Sua prática exige disciplina e postura, trabalha o auto controle, melhora o condicionamento físico e promove o ganho de massa muscular ${ }^{(1,3)}$.

Para se terum bom resultado neste esporte, é necessário um bom treinamento técnico, tático e físico ${ }^{(4)}$. O treinamento técnico é necessário para que se tenha ação certa dos golpes, sendo o mais eficiente possível ${ }^{(4)}$. O treinamento tático é realizado para que o atleta utilize os golpes na hora certa, de acordo com o seu estilo de luta e de seu adversário. O treinamento físico é para que o atleta desenvolva sua técnica com o máximo de rendimento possível. De acordo com, Barbanti (5), “O treinamento físico é uma repetição sistemática de movimentos que produzem reflexos de adaptação morfológica e funcional, com o objetivo de aumentar o rendimento num determinado espaço de tempo"(4).

A prática de muay thai requer um excelente condicionamento físico para se almejar bons resultados; para isso é necessário realizar um treinamento específico das capacidades físicas utilizadas neste esporte ${ }^{(4)}$. Através dos conhecimentos sobre as técnicas do muay thai, entendendo qual condicionamento físico e 
capacidades físicas prevalecem e quando deverão ser utilizadas em combate ${ }^{(4,6)}$.

Wilmore e Costil ${ }^{(7)}$, relatam que o corpo se adapta aos exercícios praticados regularmente por algumas semanas, e explica sobre estas adaptações: "As adaptações fisiológicas que ocorrem com a exposição crônica ao exercício melhoram tanto a sua capacidade de realizá-lo quanto a sua eficiência."

A flexibilidade é uma capacidade física reconhecida como uma das mais importantes componentes da aptidão física e que alguns testes de flexibilidade estão inseridos nas principais baterias de avaliação da aptidão física, quer associada à performance, quer à saúde. Ainda que careça de documentação objetiva que os indivíduos com maior grau de flexibilidade sejam suscetíveis a menor risco de lesão músculo ligamentar, na base desta importância está a suposição dos baixos níveis de flexibilidade estarem associados a muitas lesões ou condições crônicas, pelo que um certo grau de flexibilidade parece atuar positivamente sobre a saúde e melhoria da qualidade de vida (DE SOUZA VALE; BARRETO; DA SILVA NOVAES; DANTAS, 2006).

O presente estudo se torna relevante em razão do crescente aumento da prática de atividades físicas, mais especificamente das artes marciais como o muay thai ${ }^{(2)}$. Essa prática vem sendo desenvolvida tanto sem finalidade competitiva, sem a busca pelo máximo rendimento, buscando a contribuição na criação de hábitos de vida saudáveis que tendem a melhorar a saúde quanto por atletas cujo treinamento físico é a base para essa atividade, pois é através do treinamento que os atletas condicionam o seu corpo, aumentando sua flexibilidade, sua força, sua massa muscular, diminuem também o seu percentual de gordura e melhoram sua capacidade aeróbica e anaeróbica ${ }^{(8)}$. Desta forma, faz-se necessário o estudo das capacidades físicas, como a capacidade física potência que serão treinadas para que o indivíduo exerça o melhor de suas capacidades, alcançando assim o seu melhor potencial. Devido ao exposto o objetivo do presente estudo foi analisar as principais capacidades físicas que interferem diretamente no condicionamento de indivíduos que praticam o muay thai

Para desenvolver o objetivo se faz necessário apresentar as seguintes perguntas norteadoras deste estudo, pois, de acordo com a literatura: "Quais capacidades físicas são usadas na prática do muay thai?”; "Quais capacidades físicas interferem na performance do condicionamento físico de praticantes do Muay Thai?”.

\section{MÉTODOS}

O presente estudo se caracteriza por ter abordagem qualitativa-exploratória com ênfase na pesquisa bibliográfica, que é desenvolvida a partir de livros e artigos científicos. Em estudos originais buscou-se informações sobre praticantes de Muay Thai saudáveis e sem necessidades de adaptações no treinamento e abordagens sobre condicionamento físico e capacidades físicas. 


\section{RESULTADOS E DISCUSSÃO}

\section{O Muay Thai, sua origem e regras}

\section{atuais}

Há várias teorias com relação ao surgimento do muay thai, uma delas afirma que seu surgimento se deu através da migração do povo de AuLai para Sião, quando precisavam defender o povo de animais e ataques nômades ${ }^{(1,}$ 9). Outra teoria afirma que ele se desenvolveu já na Tailândia, quando os homens precisaram defender a conquista de suas terras ${ }^{(9)}$.

Independentemente do contexto em que surgiu, sabe-se que a arte é descendente de Myay Boran, uma luta das nove armas que marcou a história do Ocidente. Nela, os oponentes visavam aleijamento, imobilizações, estrangulamento, torções, golpes em pontos vitais e morte ${ }^{(9)}$.

Nai Khanohm Tom foi considerado o primeiro lutador da história do muay thai ${ }^{(9)}$. Em 1974, ele derrotou nove competidores ao ser capturado pelo exército birmanês e desafiado a lutar na celebração de Ket Tat Pagoda. Diz a lenda que o lutador impressionou os birmaneses ao realizar o ritual de pré-luta, a dança cerimonial chamada ram muay que homenageia o mestre e a terra na qual o combate irá se realiza ${ }^{\mathrm{r}(1)}$.

O muay thai é uma atividade predominantemente anaeróbia e o sistema do fosfagênio é o principal sistema energético depletado durante os combates, no entanto, a presença de treino aeróbio deve predominar em modalidades que durem mais de sessenta segundos ${ }^{(10-13)}$.

Seus golpes são caracterizados por grande potência e precisão, sempre com a intenção de encerrar a luta com um nocaute $(\mathrm{KO})^{(10,14)}$. Situação que justifica a realização de trabalhos físicos que desenvolvam as valências físicas dominantes que a modalidade exige, porém sem negligenciar o trabalho das capacidades que não exercem grande influência no rendimento de $\operatorname{atletas}^{(13)}$.

A CBMT, busca a isonomia em sua forma de desenvolver competições, incluindo várias categorias como regras rígidas, ou seja, existem divisões de categorias que se direcionam ao respeito das diferenças entre faixas-etárias, sexos e grau de experiência dos atletas. Tais normas visam, também, a garantia da integridade física de seus atletas ${ }^{(2)}$.

As competições são baseadas em comitês, no qual dois atletas se enfrentam trocando golpes, em chutes que geram pontuações; as lutas ocorrem em 3 rounds para amadores e 5 rounds para profissionais; cada round tem a duração de 3 minutos; a queda gera ponto para o oponente; o golpe efetuado com sucesso gera ponto para quem deferiu o golpe; ganha o atleta com maior pontuação; caso um golpe efetuado, independente da pontuação, impossibilite o adversário de continuar é anunciado knock-out e vitória para quem inferiu o golpe $\mathrm{e}^{(2)}$. 


\section{Capacidades físicas e aplicação no Muay Thai}

As capacidades físicas são as possibilidades de performance manifestadas através da ação do aparelho locomotor ${ }^{(15)}$. As capacidades físicas podem ser melhoradas a partir de processos constantes de aplicação de sobrecarga seguidas de um descanso capaz de propiciar um ambiente de recuperação total até a supercompensação ${ }^{(13)}$. Todas as capacidades físicas poder ter sua performance compreendida como o esforço máximo possível de ser apresentado ${ }^{(16)}$.

O condicionamento físico pode ser definido como a capacidade que cada indivíduo possui para realizar atividades físicas, podendo estar relacionada a fatores genéticos, ao estado de saúde, dos níveis de nutrição e, principalmente da prática regular de atividades físicas. Agilidade, equilíbrio, velocidade, coordenação, flexibilidade, força muscular, entre outros componentes são descritos como importantes indicadores $\mathrm{da}$ capacidade motora em crianças e adolescentes ${ }^{(17)}$.

Como aborda MAGILL ${ }^{(18)}$, é importante considerar que tais capacidades têm caráter relativamente independentes, pois testes indicativos de uma capacidade não revela informações precisas sobre outra. MAGILL ${ }^{(18)}$, ainda revela que esta lista de capacidades, de forma alguma, constitui uma totalidade de capacidades necessárias ao desempenho atlético. “... não se pode supor que as listas derivadas desses testes incluam todas as capacidades motoras humanas. Existem outras tarefas motoras que também deveriam ser incluídas nestas listas, para elas se constituírem em listas completas." (MAGILL, 1984, pg. 160).

O condicionamento físico dos praticantes do muay thai considera o desenvolvimento das capacidades de forma geral e específica. Nesta visão o treinamento físico é de grande necessidade e importância, pois ele influenciará na força, velocidade, flexibilidade e no alcance de resistência do atleta, que está diretamente ligada ao seu desenvolvimento técnico e tático. De acordo com Rosa ${ }^{(8)}$, as capacidades físicas agilidade, flexibilidade e força pré-treinamento sistematizados, apresentam diferenças significativas no pós-treinamento. No muay thai a força, agilidade de movimento, estímulo e flexibilidade são de grande relevância para resistir o esgotamento físico e a aplicação dos golpes, além de impedir lesões tanto no treino como na hora do confronto ${ }^{(8)}$.

Zakharov e Gomes ${ }^{(19)}$, citam que a resistência enquanto capacidade física "caracteriza as possibilidades do desportista de realizar, durante um tempo prolongado, o trabalho muscular, mantendo os parâmetros dados de movimento".

\section{Agilidade}

Segundo Gallahue(20), agilidade é a habilidade de alterar a direção do corpo, rápida e precisamente. Com ela pode-se fazer alterações, rápidas e precisas, na posição do corpo, durante o movimento. 
Para Oliveira ${ }^{(21)}$, a agilidade é uma variável neuro-motora caracterizada pela capacidade de realizar trocas rápidas de direção, sentido e deslocamento da altura do centro de gravidade de todo corpo ou parte dela. A agilidade no âmbito esportivo é a resposta do indivíduo em conseguir executar movimentos rápidos e coordenados dentro de especificidade técnica ${ }^{(21)}$.

Quando trabalhamos agilidade, desenvolvemos o domínio do corpo e a confiança em si. Para um perfeito desenvolvimento da agilidade, devemos apresentar um progressivo grau de dificuldade nos exercícios, sendo que com esta programação, podemos sentir que os atletas alcançarão níveis ótimos de execução ${ }^{(21)}$.

Em um combate é exigido do atleta que execute inúmeros ataques, defesas, contragolpes e esquivas de forma sistemática e em um ritmo e momento apropriado, normalmente são executados ataques e bloqueios simultâneos ou em ataques em resposta aos estímulos do adversário e ainda são utilizados ataques combinados que utilizam lados opostos do corpo demandando grande agilidade, que aqui já foi definida por Carnaval ${ }^{(22)}$ como a capacidade que o indivíduo tem de realizar movimentos rápidos com mudança de direção e sentido. Nas categorias mais leves é muito mais intensa a aplicação dessa valência, já nas categorias de peso mais altas é menos observada, porém não menos importante no combate.

\section{Flexibilidade}

A flexibilidade é uma capacidade física reconhecida como uma das mais importantes componentes da aptidão física e que alguns testes de flexibilidade estão inseridos nas principais baterias de avaliação da aptidão física, quer associada à performance, quer à saúde ${ }^{(23)}$.

Alguns autores sugerem que níveis reduzidos de flexibilidade refletem em um suposto aumento do nível de susceptibilidade do atleta a lesões mais graves ${ }^{(23)}$.

Além do desenvolvimento da flexibilidade ser pré-requisito no desempenho de habilidades que requerem amplitudes exageradas, o atleta pode aprimorar a capacidade de executar movimentos rápidos. Fundamentalmente a componente flexibilidade tem íntima ligação não só com o alongamento muscular, mas também está diretamente relacionada com a elasticidade de ligamentos e tendões ${ }^{(13,23)}$.

A flexibilidade é uma capacidade física reconhecida como uma das mais importantes componentes da aptidão física e que alguns testes de flexibilidade estão inseridos nas principais baterias de avaliação da aptidão física, quer associada à performance, quer à saúde ${ }^{(24)}$. Ainda que careça de documentação objetiva que os indivíduos com maior grau de flexibilidade sejam suscetíveis a menor risco de lesão músculo ligamentar, na base desta importância está a suposição dos baixos níveis de flexibilidade estarem associados a muitas lesões ou condições crônicas, pelo que um certo grau de flexibilidade 
parece atuar positivamente sobre a saúde e melhoria da qualidade de vida ${ }^{(24)}$.

A flexibilidade consiste na qualidade física que permite ao indivíduo executar um movimento de grande amplitude músculo-articular sem representar riscos de lesões ${ }^{(25)}$. Pode ser treinada e acrescentar benefícios ao corpo em crianças, jovens, adultos e idosos. Para que as crianças atinjam um desenvolvimento motor harmonioso, a flexibilidade é imprescindível pela necessidade de ajustes posturais no período de maior crescimento, que exigem o equilíbrio nas cadeias musculares anteriores e posteriores, responsáveis pelo equilíbrio do corpo e sustentação da coluna vertebral ${ }^{(25)}$.

No grupo do Muay Thai, notamos associação direta entre a altura com a flexibilidade, atletas com estatura inferior aos seus oponentes necessitam de grandes amplitudes de movimentos para suprir sua estatura no momento do gesto esportivo. Neste grupo, não foram encontrados estudos que avaliam a flexibilidade em atletas da modalidade, porém desde 1979 Barbanti, classifica a flexibilidade como de extrema importância nesse esporte, pois aumenta o rendimento esportivo do atleta. Evidenciando assim a importância de profissionais como fisioterapeutas para auxiliar no ganho e na manutenção dessa habilidade.

\section{Força e Potência}

A força dos atletas se torna um item indispensável para um resultado satisfatório em competições em relação à contundência dos golpes, sendo observado no estudo uma melhora significativa na força dos atletas/praticantes no pós-treino, indicando necessidade de trabalho específico antes do combate visando otimizar essa qualidade, e infere-se a possibilidade de contribuição do treinamento de muay thai para o desenvolvimento da força, em especial nos membros superiores, no grupo estudado(26).

Uma definição precisa de força levando em conta os seus aspectos físicos e psíquicos representa uma grande dificuldade, uma vez que o tipo de força, o trabalho muscular, os diferentes caracteres do trabalho muscular são influenciados por muitos fatores ${ }^{(26)}$. A força é a habilidade de um músculo ou grupo muscular de vencer uma resistência produzindo tensão na ação de empurrar tracionar ou elevar ${ }^{(27)}$.

Segundo Barbanti $^{(5)}$, no esporte e na atividade física, a força motora manifesta-se no aparelho locomotor, dependendo do sistema nervoso que o dirige, do sistema ósseo que o sustenta e dos sistemas cardiovasculares e respiratório que transportam os nutrientes necessários para o desenvolvimento de sua tarefa. Portanto do ponto de vista prático a força motora é a capacidade do sistema neuromuscular de vencer resistências (oposições), como por exemplo o peso do próprio corpo, um peso, um objeto, etc. Meusel (1969 apud BARBANTI, 2001) apresentou uma conceituação bastante clara e objetiva sobre força, segundo o autor "é uma característica humana, com qual move-se 
uma massa (seu próprio corpo ou um implemento esportivo), sua capacidade em dominar ou reagir a uma resistência pela ação muscular”.

\section{CONSIDERAÇÕES FINAIS}

Para um correto treinamento devese conhecer o esporte praticado, analisando-o para construir um planejamento de treinamento com a aplicação das sobrecargas especificas e necessárias aos objetivos do treinamento, principalmente quanto aos sistemas energéticos predominantes e as valências físicas utilizadas durante a pratica da atividade. $\mathrm{O}$ planejamento do treinamento desportivo pautado no princípio da especificidade está diretamente ligado ao treinamento das valências físicas. Para uma melhor preparação física devem-se detectar as valências físicas intervenientes do esporte a ser realizado, e treinar especificamente estas valências físicas utilizadas neste esporte. Esta análise está diretamente ligada ao princípio da especificidade do treinamento. O sistema de luta do muay thai é muito parecido com o do boxe, pois o combate é realizado em locais semelhantes (ringues iguais), e a mesma vestimenta (calção, luva e coquilha), com exceção da sapatilha usada no boxe. A principal diferença entre o muay thai e o boxe tradicional é que no muay thai são permitidos ataques com os membros inferiores (chutes e joelhadas), e em algumas competições, cotoveladas. O muay thai é semelhante ao boxe amador, por apresentar o tempo de luta e a quantidade de rounds similares. A resistência de força rápida no muay thai é utilizada em quase todas as técnicas, pois elas se utilizam da velocidade de movimento e deve atingir forte o adversário, força rápida (potência). Concluindo, a principal valência física utilizada para atacar e golpear o adversário é a resistência de força rápida, utilizadas tanto pelos membros superiores (socos e cotoveladas) quanto pelos inferiores (chutes e joelhadas). A agilidade é a valência que no muay thai faz com que as sequências, os golpes e defesas sejam aplicados com o máximo de força e velocidade com uma técnica perfeita. A agilidade é utilizada pelos movimentos dos membros superiores e inferiores, e ainda pela combinação de golpes destes segmentos. Quanto à flexibilidade de membros superiores e de tronco são secundárias, e a flexibilidade de membros inferiores são mais importantes. No muay thai a flexibilidade de membros inferiores é utilizada principalmente nos chutes altos, (chutes na altura da costela e na altura da cabeça). A s s i m diante desse contexto que foi apresentado, dentro da literatura pesquisada e dos artigos encontrados, fica claro para nós que o muay thai interfere sim de forma positiva nas capacidades físicas melhorando-as de forma significativa, desde que se tenha um programa de treinamento bem elaborado, utilizando às valências físicas de forma específica da modalidade, e assim melhorando o condicionamento físico. 


\section{REFERÊNCIAS}

1. Müller Júnior IL, Capraro AM. A warrior identity forged based on knee and elbow strikes: the narratives of the first masters of Brazilian muay thai. Revista de Artes Marciales Asiáticas. 2020;15(1):22-33.

2. CBMT CBdMT. História do Muay Thai 2021 [Available from: https://cbmt.com.br/historia/.

3. Pessina JE. Gênero no Muay Thai: uma luta dentro das artes marciais. 2017.

4. Souza FB, Farje LF, editors. Elementos Fundamentais para um Treinamento Eficaz em Atletas de Muay Thai. VIII JORNACITEC-Jornada Científica e Tecnológica; 2019.

5. Barbanti V. Treinamento Físico. $3^{\mathrm{a}}$ edição. Balieiro; 2001.

6. Silveira D. Guia básico de treinamento Muay Funcional: Autografia; 2019.

$7 . \quad$ Wilmore JH, Costill DL. Physical energy: fuel metabolism. Nutrition reviews. 2001;59(1):S13.

8. Rosa GJd. Análise de valências físicas em lutadores de muay thai: Universidade Tecnológica Federal do Paraná; 2015.

9. Paiva L. Olhar Clínico nas Lutas, Artes Marciais e Modalidades de Combate: Preparação Física-HistóriaAntropologia-Psicologia-Nutrição-Sociologia-Medicina Esportiva: OMP EDITORA; 2015.

10. Podhurskyi SE, Pavlenko IA. Differentiated approach to the development of speed-strength capabilities of qualified Muay-Thai athletes, taking into account weight categories. Retos: nuevas tendencias en educación física, deporte y recreación. 2021(40):5.

11. Tagliani ALDSG. Muay Thai: uma estratégia de treinamento físico para obesos no Brasil. 2020.

12. Ribas MR, Pereira E, Borato LZ, da Silva Damasceno M, Almeida LV, Bassan JC. Indicadores antropométricos de potência e capacidade anaerobia em atletas de taekwondo. RBPFEX-Revista Brasileira De Prescrição E Fisiologia Do Exercício. 2020;14(89):12230.

13. Bompa TO. Periodizaçao No Treinamento Esportivo, a: Editora Manole Ltda; 2001.

14. dos Santos DE, Diniz EFFS, Lavorato VN, de Oliveira RAR. APTIDÃO FÍSICA EM PRATICANTES DE MUAY THAI DO SEXO FEMININO. Caderno Científico FAGOC de Graduação e Pós-Graduação. 2020;4(2).

15. Vaca García MR, Gómez Nicolalde RV, Cosme Arias FD, Mena Pila FM, Yandún Yalamá SV, Realpe Zambrano ZE. Estudio comparativo de las capacidades físicas del adulto mayor: rango etario vs actividad física. Revista Cubana de Investigaciones Biomédicas. 2017;36(1):1-11.

16. Simões R, Moreira WW, Pellegrinotti IL. Performance do atleta: reflexões e percepções sobre o corpo. Revista Brasileira de Ciência e Movimento. 2017;25(2):62-72.

17. Bergmann MLdA, Halpern R, Bergmann GG. Perfil lipídico, de aptidão cardiorrespiratória, e de composição corporal de uma amostra de dscolares de $8^{a}$ série de Canoas/RS. Revista Brasileira de Medicina do Esporte. 2008;14(1):22-7.

18. Magill RA. Aprendizagem motora: conceitos e aplicações: Editora Blucher; 1984.
19. Zakharov AA, Gomes A. Ciência do Treinamento desportivo: aspectos teóricos e práticos da preparação do desportista, organização e planejamento do processo do treino: controle da preparação do desportista. Organização e adaptação de Antonio Carlos Gomes Rio de Janeiro, RJ Ed Palestra Sport. 2003.

20. Gallahue DL, Donnelly FC. Developmental physical education for all children: Human Kinetics; 2007.

21. Oliveira M. Influência do ritmo na agilidade em futebol. 2000, 107f: Dissertação (Mestrado em Educação Física)-Universidade Federal de São Paulo ...; 2000.

22. Carnaval ACOdQ. Aspectos da morfologia e biologia reprodutiva de uma população de Scinax argyreornatus em Majé, no Estado do Rio de Janeiro (Amphibia, Anura, Hylidae). 1997.

23. Lima BN, Lucareli PR, Gomes WA, Silva JJ, Bley AS, Hartigan EH, et al. The acute effects of unilateral ankle plantar flexors static-stretching on postural sway and gastrocnemius muscle activity during single-leg balance tasks. Journal of sports science \& medicine. 2014;13(3):564.

24. Souza Vale RGd, Barreto ACG, Silva Novaes Jd, Dantas EHM. Efeitos do treinamento resistido na força máxima, na flexibilidade e na autonomia funcional de mulheres idosas. Rev bras cineantropom desempenho hum. 2006;8(4):52-8.

25. Ferreira J, Ledesma N. Indicadores de flexibilidade em escolares de 11 anos de idade de uma escola de Campo Grande-MS, Brasil. Revista Digital efdeportes Buenos Aires Ano. 2008;12.

26. Fleck SJ, Kraemer WJ. Fundamentos do treinamento de força muscular: Artmed Editora; 2017.

27. Oliveira Santos ECd, Galvão LL, Tribess S, Meneguci J, Santos RGd, Silva RR, et al. Valores normativos de força muscular em idosos. Arquivos de Ciências do Esporte. 2019;6(4).

OBSERVAÇÃO: Os autores declaram não existir conflitos de interesse de qualquer natureza. 